Preprint typeset in JINST style - HYPER VERSION

\title{
Testing of Cryogenic Photomultiplier Tubes for the MicroBooNE Experiment
}

\author{
T. Briese $^{d}$, L. Bugel ${ }^{b}$, J. M. Conrad ${ }^{b}$, M. Fournier ${ }^{d}$, C. Ignarra ${ }^{b}$, B. J. P. Jones ${ }^{b}$, \\ T. Katori ${ }^{b}$, R. Navarrete-Perez ${ }^{c *}$ P. Nienaber ${ }^{d}$, T. McDonald $^{d}$, B. Musolf ${ }^{e}$, A. Prakash ${ }^{b \dagger}$ \\ E. Shockley ${ }^{d}$, T. Smidt ${ }^{b}$, K. Swanson ${ }^{a}$, M. Toups ${ }^{b}$ \\ ${ }^{a}$ Physics Department, California Institute of Technology, Pasadena, CA 91125 \\ ${ }^{b}$ Physics Department, Massachusetts Institute of Technology, Cambridge, MA 02139 \\ ${ }^{c}$ Instituto de Ciencias Nucleares, Universidad Nacional Autónoma de México, D.F. 04510, \\ México \\ ${ }^{d}$ Physics Department, Saint Mary's University of Minnesota, Winona, MN 55987 \\ ${ }^{e}$ Warren Township High School, Gurnee, IL 60031 \\ E-mail: KATORI@FNAL.GOV
}

\begin{abstract}
The MicroBooNE detector, to be located on axis in the Booster Neutrino Beamline (BNB) at the Fermi National Accelerator Laboratory (Fermilab), consists of two main components: a large liquid argon time projection chamber (LArTPC), and a light collection system. Thirty 8inch diameter Hamamatsu R5912-02mod cryogenic photomultiplier tubes (PMTs) will detect the scintillation light generated in the liquid argon (LAr). This article first describes the MicroBooNE PMT performance test procedures, including how the light collection system functions in the detector, and the design of the PMT base. The design of the cryogenic test stand is then discussed, and finally the results of the cryogenic tests are reported.
\end{abstract}

KEYWORDS: MicroBooNE, Liquid argon, cryogenic photomultiplier tube.

\footnotetext{
*Present address: Astronomy and Astrophysics Department, University of California, Santa Cruz, 95064

${ }^{\dagger}$ Present address: Physics Department, The Ohio State University, Columbus, 43210
} 


\section{Contents}

1. Introduction 1

1.1 The MicroBooNE experiment at Fermilab 1

1.2 The light collection system 2

1.3 The PMT unit 2

1.4 The PMT base design 3

2. PMT test stand 5

2.1 PMT test for MicroBooNE

2.2 PMT test stand design 5

2.3 Test procedure 5

3. Results

3.1 Special tests 6

3.1.1 Stability test 7

3.1.2 Gain change with cooling time 8

3.1.3 Linearity test 8

3.1.4 Rate dependence of PMT gain 10

3.2 MicroBooNE PMT gain measurement 11

3.3 MicroBooNE PMT dark current measurement 13

4. Conclusion 14

\section{Introduction}

\subsection{The MicroBooNE experiment at Fermilab}

The MicroBooNE detector is a LArTPC positioned in the BNB at the Fermilab, scheduled to begin data collection in 2014 [1]. It is a milestone experiment on the path to future large LArTPC detectors for long baseline neutrino oscillation experiments, such as LBNE [2], LBNO [3], and Okinoshima [ $\llbracket$; it is expected that MicroBooNE will provide important design and construction information for these experiments. The 170 tons of liquid argon of the MicroBooNE cryostat contains $2.5 \times 2.4 \times 10.4 \mathrm{~m}^{3}$ TPC drift volume. Thirty cryogenic 8 -inch PMTs are located behind the wire planes to observe the argon scintillation light following the basic design of the ICARUS T600 detector [5]. 


\subsection{The light collection system}

The scintillation light from a minimum ionizing particle (MIP) is roughly 40,000 photons/MeV [6]. The presence of electric field ( $500 \mathrm{~V} / \mathrm{cm}$ for MicroBooNE) reduces this to $\sim 60 \%$ [7]. Among them, $30 \%$ of light corresponds to the prompt light [8] and useful to trigger the TPC. Thus, a $1 \mathrm{MeV}$ MIP in the MicroBooNE TPC volume emits $\sim 7200$ prompt photons.

The light collection system of MicroBooNE consists of 32 8-inch PMTs. Large photocathode PMTs provide a cost-effective solution to maximize photocathode coverage; this design yields a $0.9 \%$ photocathode coverage. By this choice, conservatively, PMTs with $\sim 1 \%$ total efficiency (including PMT quantum efficiency, wavelength shifter efficiency, and geometric loss), can trigger, say, a $10 \mathrm{MeV}$ MIP.

The very important feature of the light collection system is its ability to measure the event time at the $\sim$ ns level. Since prompt light from the liquid argon is emitted on a 3-6 ns time scale, detection of the scintillation light allows the LArTPC to be triggered, where drift of electrons take $\sim \mathrm{ms}$. Although MicroBooNE is an accelerator-based neutrino beam experiment and trigger information can be obtained from the accelerator clock via the Fermilab ACNET (accelerator network), there are a number of reasons that the light collection system is useful. First, any non-beam related physics, such as supernova neutrinos, requires a trigger from the light collection system. Second, scintillation light information can distinguish physics events from cosmic ray backgrounds via timing. MicroBooNE will run on the surface, and $\sim \mathrm{kHz}$ rates of cosmic ray events in the drift volume are anticipated. The probability that cosmic rays will overlap with the BNB beam pulse $(1.6 \mu \mathrm{s})$ is low, but any cosmic rays before or after the beam pulse still will leave tracks in the TPC if those rays pass during the drift time $(\sim \mathrm{ms})$, causing a confusion in the event matching. Third, scintillation light itself is a useful measure of the total energy deposited by an event. Finally, the pulse shape information from the scintillation light could be used for particle identification.

\subsection{The PMT unit}

Each PMT unit is defined by a PMT in the PMT mount (Teflon mount fixed by spring loaded Teflon coated wires) fitted with a wavelength shifter deposited acrylic plate and a magnetic shield. Figure 1] shows the prototype of the MicroBooNE PMT unit.

The light collection system uses 8-inch diameter, bi-alkali photocathode Hamamatsu R591202mod cryogenic PMTs [9]. The R5912-02mod is a 14-stage high gain PMT (109 gain with the average optimal voltage $\sim 1700 \mathrm{~V}$ ); the intrinsically high gain of the tube helps to compensate for the diminution of the gain in the cryogenic environment. This also permits operation at a slightly lower voltage, which avoids high voltage (HV) breakdown on the base, the cable connection, or the feed-through. The bi-alkali photocathode stops working below $150 \mathrm{~K}$ [10]. This is cured by adding a thin platinum layer between the photocathode and the borosilicate glass envelope to preserve the conductance of the photocathode at low temperature. The large photo-cathode and high gain makes R5912-02mod a suitable choice for us.

Since the argon scintillation light is in the vacuum UV region $(128 \mathrm{~nm})$ and thus cannot penetrate glass, tetra-phenylbutadiene (TPB) is used as a wavelength shifter. A TPB-polystyrene mixture is deposited on an acrylic plate positioned in front of each PMT [11]. The degradation of TPB, particularly by the exposure to UV light, has been carefully studied [12, 13]. 


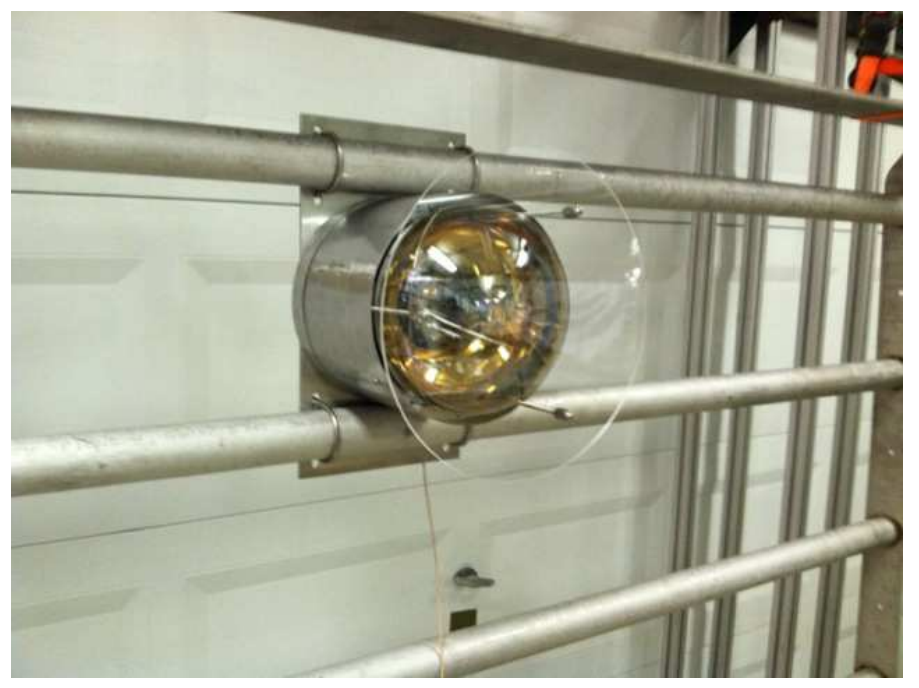

Figure 1. A picture of the mechanical model of the MicroBooNE PMT unit in the test facility. A PMT is fixed in the mount, which is surrounded by the magnetic shield up to the equator of the PMT, and the wave length shifter deposited acrylic plate is equipped in front of the PMT.

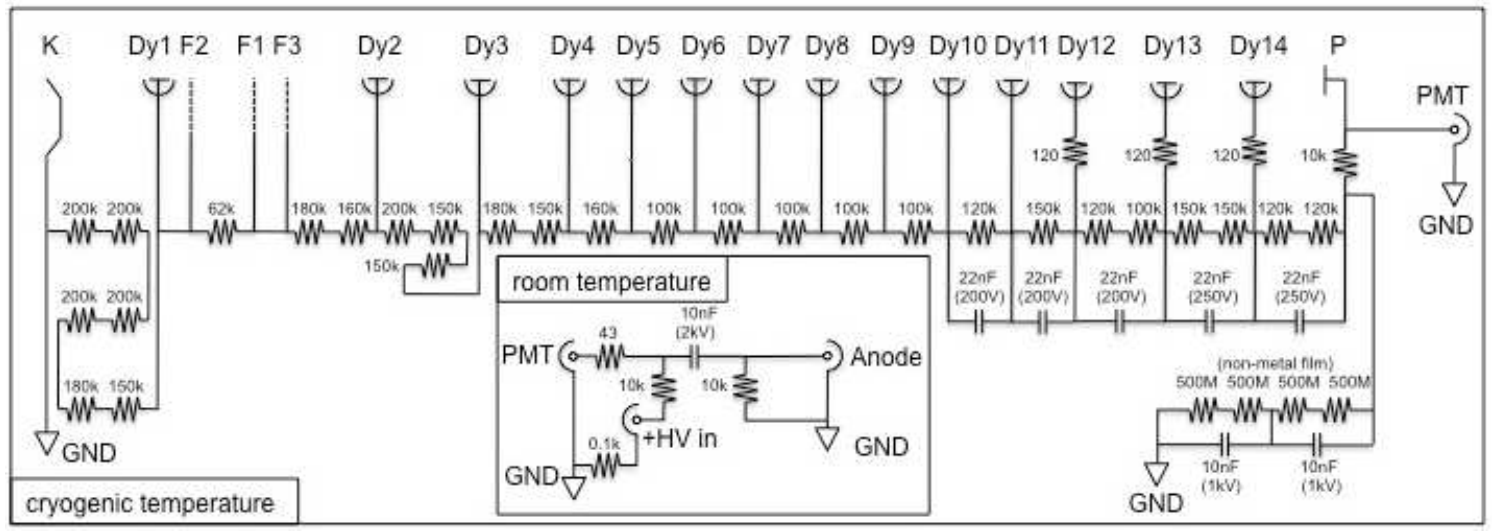

Figure 2. The schematic of the MicroBooNE PMT. PMTs and bases are both located inside the cryostat at cryogenic LAr temperature. Since the PMT is operated with positive HV, a decoupling capacitor is required to split the signal from the HV outside of the cryostat (that is, at room temperature).

The performance of such a large diameter PMT is sensitive to the Earth's magnetic field and a cryogenic magnetic shield has been designed and tested. It is made of a material featured with nonzero permeability at cryogenic temperatures [14]. Our tests show that these specially made shields are effective at liquid nitrogen temperatures, and shielding performance, especially with respect to the orientation of the PMT in the earth magnetic shield, is consistent with other published results [15].

\subsection{The PMT base design}

The base is designed for positive HV operation, i.e., the photocathode is grounded, and the last dynode in the chain is held at high voltage. This scheme has a number of advantages for present purposes. First, only one cable is needed for each channel, as each cable carries both the DC HV 


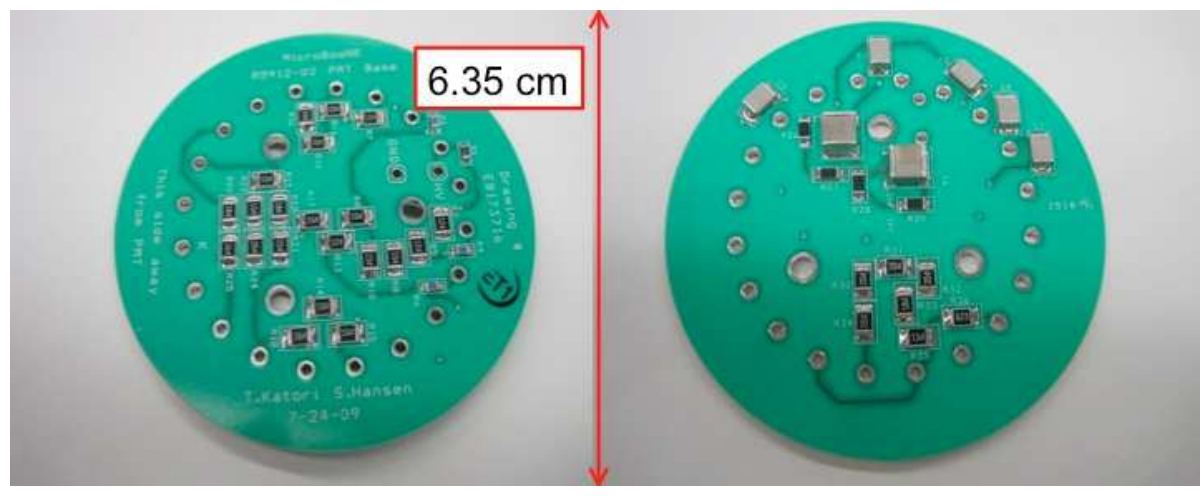

Figure 3. Pictures of the MicroBooNE PMT base.

and the signal. This reduces the number of penetrations of the cryostat, which often require special structures. Second, grounding the photocathode reduces noise, which helps with the detection of low energy events. Third, a grounded photocathode will not interfere with the TPC. Since the PMTs are located right behind the TPC anode wires, a nonzero potential at the PMT photocathode would alter the TPC electric field. For MicroBooNE, the last collection plane is grounded. A grounded photocathode will therefore not affect the field in the TPC field cage. Finally, grounding the photocathode makes for a good electric field inside the PMT. When the photocathode is not grounded, the tube requires a grounded shield, which is cumbersome for the large diameter PMT MicroBooNE uses.

Figure 2 shows the schematic of the MicroBooNE PMT base. The dynode chain follows the recommended resistance ratios from the manufacturer [9]. Several features were added to this basic design. A charge "reservoir" at the last stage is provided by the large capacitors through the ground, and this reservoir supplies electrons quickly to the later dynodes. Since PMT is operated with positive high voltage, the signal needs to be split from the HV; this happens outside the cryostat, by means of a room temperature splitter circuit.

Figure 3 shows a picture of the MicroBooNE PMT base. Special attention is paid to the choice of passive components. All passive components are surface mounted, and only metal film resistors and $\mathrm{C} 0 \mathrm{G} / \mathrm{NP} 0$ type capacitors, known to have the smallest temperature dependencies, are used. Materials for the PC board and the cable are also chosen carefully to minimize water and oxygen contamination, and to perform at cryogenic temperatures. The PC board is made from Roger4000 series [16], and the cable has an FEP outer jacket and PTFE inner insulator.

All bases were tested after the production and were then soldered to a PMT. No thermal contraction caused base failures have been observed. A similar base was designed for a 2-inch head-on Hamamatsu R7725-mod cryogenic PMT and used in R\&D studies at the Massachusetts Institute of Technology (MIT) [17]. The MIT group observed several base failures due to the failure of the $10 \mathrm{k} \Omega$ resistor at the end of the dynode chain. Damage to this component appears to have been caused by the impulse current generated by the splitter shorting in the gaseous phase argon. 


\section{PMT test stand}

\subsection{PMT test for MicroBooNE}

Thirty-three (30 plus 3 spare) 8-inch MicroBooNE PMTs were tested both in air (room temperature) and in liquid nitrogen (LN2, cryogenic temperature). Here the test in the LN2 (77 K) represent the MicroBooNE environment, where all apparatus is immersed in the liquid argon (87 K). The main purpose of this test was to identify possibly defective PMT assemblies in a cryogenic environment before installing them in the cryostat. Two main parameters were measured: the gain and the dark current. We found all of the PMTs performed properly at cryogenic temperature. A sample of the PMTs were selected for specific additional tests.

\subsection{PMT test stand design}

Figure 7 shows a drawing of the PMT test stand. The stand is based on a $346 \mathrm{~L}$ open Dewar [18], which can be filled either with LN2 or with room-temperature air. The Styrofoam and glass fiber Dewar lid is modified to include five penetrations: a LN2 filling line, a vent for gaseous nitrogen from boil-off, a LN2 level-monitor probe, a cable feed-through, and a light injection system to carry measured amounts of light into the otherwise light-tight interior. The cable feed-through is a commercial four-connector SHV vacuum feed-through [19], with a custom modification to prevent high-voltage breakdown (allowing the connector to be used in gaseous argon). An optical fiber runs inside a metal fixture passing through the center of the lid. The exterior end of the fiber is exposed to light from a pulsed blue LED and the end of the fiber inside the Dewar is optically coupled to a small sand-blasted glass piece which diffuses the light so as to illuminate the PMTs under test. Four PMTs are held within a Delrin fixture, which is attached to the underside of the lid by a stainless steel mount. The PMTs sit on the bottom Delrin fixture during room temperature running, and tubes fit into the openings of the top Delrin fixture by the buoyancy during cryogenic running. This configuration avoids putting pressure on the PMTs by thermal contraction of the fixture.

\subsection{Test procedure}

In cryogenic running, the PMTs are immersed in liquid nitrogen, and kept in a dark environment for at least three days before any tests are performed, with the exception of the cooling test (Sec. 3.1.2). One calibration/control PMT is located at the same position in the test stand for all of the PMT tests. So as not to disturb the experimental environment, LN2 is not added to the dewar during the data taking period. The tubes are illuminated by the fiber-connected diffuser above the liquid surface. The pulser circuit and the LED are located sufficiently far away from the cryogenics to avoid temperature-dependent performance changes The LED pulser frequency is set to $10 \mathrm{~Hz}$ for all measurements except the rate dependence test (Sec. 3.1.4). LED intensity is controlled by the circuit bias voltage; the intensity is not absolutely calibrated and the absolute intensity is unknown. However, the number of photoelectrons (PEs) from the photocathode can be determined from the data, either from the Gaussian approximated Poisson distribution or the single photoelectron response (SER) spectrum depending on the light intensity. Using this information, the PMT gains are measured. The Tektronix TDS-5054B Digital Oscilloscope [20] was used for data acquisition, 


\section{PMT TEST STAND}

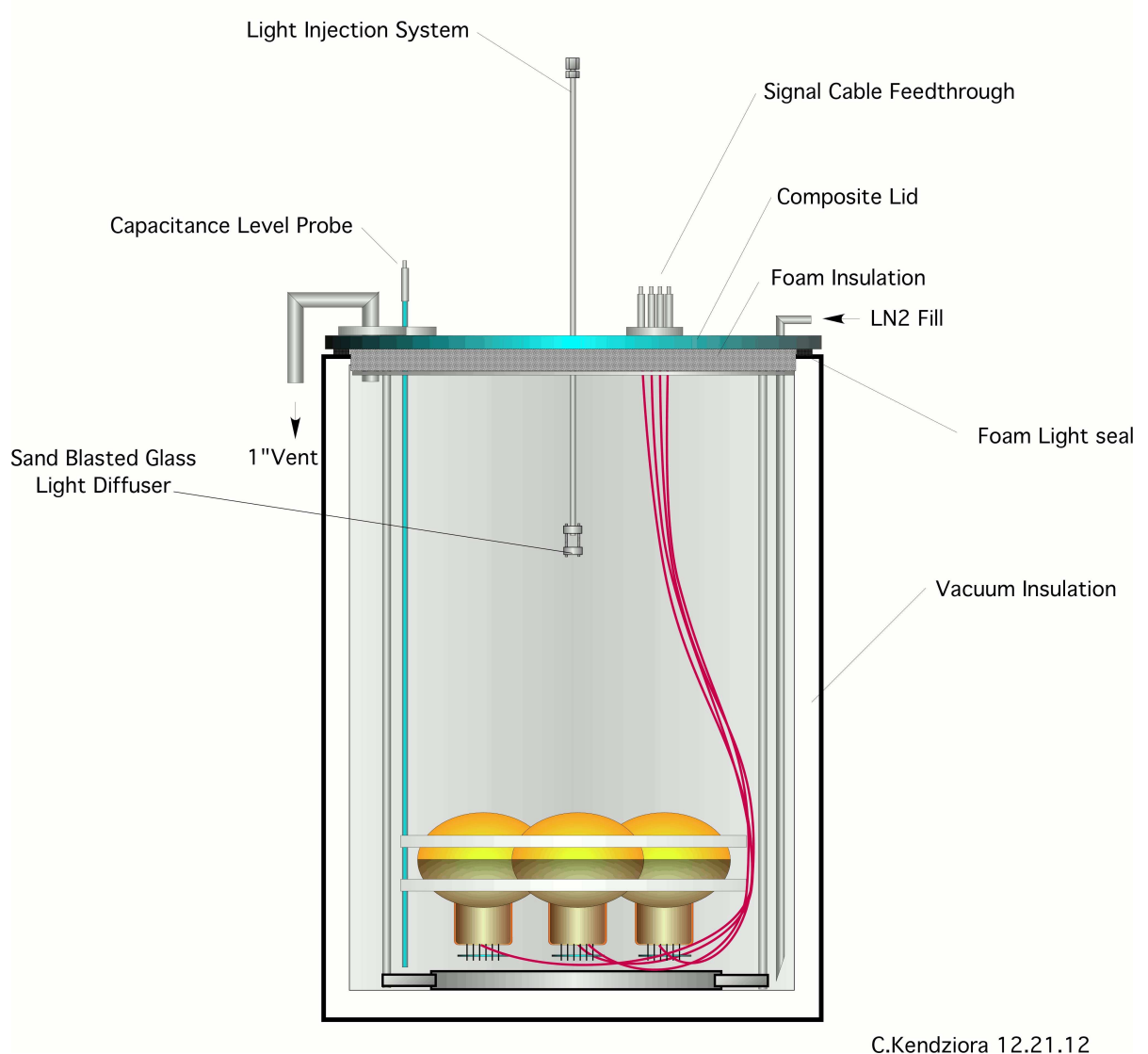

Figure 4. The PMT test stand. Four PMTs are held at the bottom of the stand; they are simultaneously tested by exposure to light from the optical fiber assembly.

and a Dzero high voltage power supply with the CSS monitoring system [21] was used to provide and monitor the PMT high voltage.

\section{Results}

\subsection{Special tests}

Selected PMTs are tested with a relatively high number (5 - 10) of PEs to check a number of tube properties. If the LED intensity is high, we first find the number of PEs through the Poisson distribution of the charge; the gain of the PMT is obtained from the averaged charge. Figure 5 shows the typical plot of this method. The mean and the variance are read from the symmetric Poisson distribution of the charge (blue histogram in Fig. 5), and the square of the ratio allows the number of PEs to be extracted. From the variation in the number of measured PEs, we estimate a $10 \%$ error on this PE measurement. This gives $12 \%$ error to the gain determination by this method. This approach is affected by any environmental effects (reflection of light inside the Dewar, for example) that broaden the charge distribution. As a result, even though this method does not 


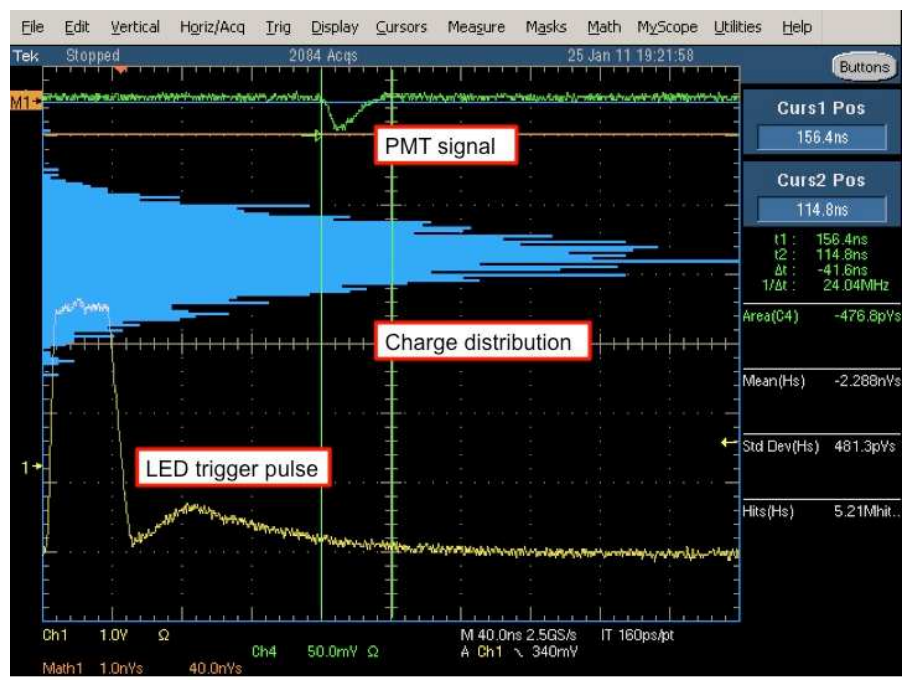

Figure 5. A typical screen shot from the TDS-5054B oscilloscope. The upper green trace shows the split signal pulse from the PMT, and the lower yellow trace is the trigger pulse for the LED. Only the rising edge of the trigger pulse is important to trigger the LED; the LED pulse is $\sim 10 \mathrm{~ns}$, shorter than the trigger pulse. The blue histogram rotated $90^{\circ}$ displays the distribution of the integrated PMT signal which measures the charge output from the PMT; this makes a symmetric Poisson distribution when light intensity is high enough ( $>5$ PEs).

involve any fit calculations and therefore yields a quick result, it is not considered to be as accurate as the gain calculated from fitting the single electron response described below (Sec. 3.2).

\subsubsection{Stability test}

This test examines the stability of the photomultiplier gain by quickly raising the tube voltage from zero to operating voltage while the PMT is at cryogenic temperature. First, three PMTs are immersed in LN2 for more than three days, during which time the tubes are kept in a light-tight environment with no applied HV. The pulsed LED illumined the tubes at low intensity, with each PMT receiving sufficient light to generate $\sim 5$ PEs. The tube voltage was then quickly ramped to an operating level where all the tubes had roughly the same gain $\left(\sim 10^{7}\right)$. Figure 6 shows the result of three PMTs measured simultaneously for this test. The upper plot shows the variation in the number of measured PEs as a function of a time. As noted above, the number of PEs is assigned a $10 \%$ error, which covers the observed variation. The number of measured PEs varies slightly with the location of the PMT in the fixture, but the number is stable for each tube over the time period shown. This stability demonstrates that the test environment does not change appreciably on this time scale, including both the liquid nitrogen level (changes in which would alter light propagation inside the Dewar) and the LED pulser system (especially the LED intensity).

The lower plot in Figure 6 shows the variation in the PMT gains. The gains change quite a bit in the first $\sim 30 \mathrm{~min}$, but after that, they are stable. This observation confirms that changing the PMT voltage while the tubes are at cryogenic temperatures does not have any particular effect on tube performance. 

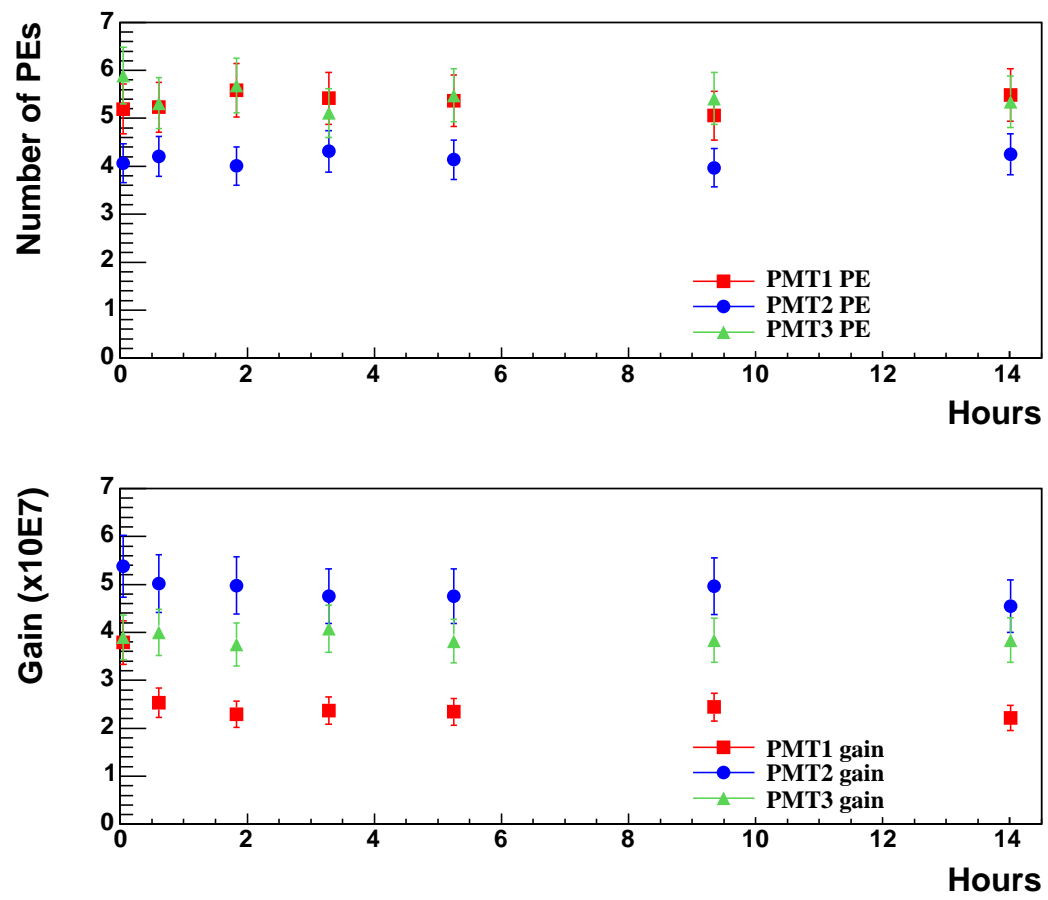

Figure 6. PMT gain measurement stability test. High voltage is suddenly applied to cooled PMTs. The upper plots show the measured number of PEs; this demonstrates the long time stability of the environment. The lower plot shows measured gain. There is some anomalous behavior in the first $\sim 30$ min, but the gain is stable thereafter.

\subsubsection{Gain change with cooling time}

The next gain stability test examines the variations when the PMT is not initially cooled for several days. Figure 7 shows the results of this study; in this case, the tube is brought up to operating voltage immediately after LN2 immersion. As with the previous test, data for three PMTs are collected simultaneously to check consistency. Data taking starts after the initial post-immersion boiling of the LN2 subsides ( $\sim 15 \mathrm{~min})$. Note the structure of the test stand does not permit keeping PMTs completely in a dark environment before immersion; as a result, the data show the combination of two effects: the actual PMT cooling, and the excitation of the photocathode due to pre-immersion light exposure. This makes it difficult to interpret the drift of measured PEs. Despite this effect, however, the dramatic change in PMT gain can clearly be observed. The PMT gain remains high in the first $\sim 5$ hours after immersion, and then suddenly drops by more than a factor of two, coming to a stable value with a small drift. This effect is attributed to the tube's interior coming to thermal equilibrium; to insure that the tube is completely cooled, all other tests described in this note will immerse the tubes for three days before proceeding.

\subsubsection{Linearity test}

This section details how the PMT response varies as a function of the LED intensity; this effect is tested using several PMTs. The LED output is first monitored by a PMT set to low gain, in order to find the dynamic range of the LED. When the LED bias voltage is 0 to $20 \mathrm{~V}$, the LED intensity 

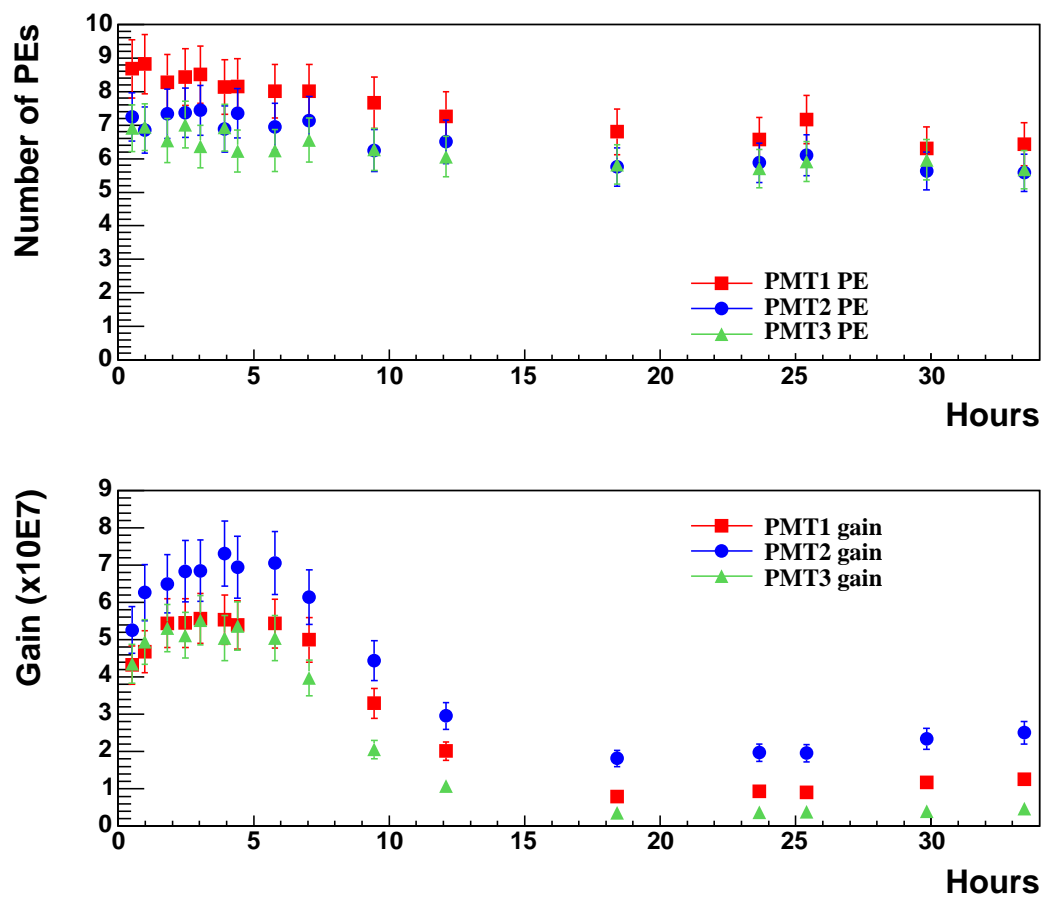

Figure 7. PMT cooling test. The drift in the number of measured PEs suggests that the photocathodes are excited before the measurement by exposure to ambient light. The bottom plot shows the gain variation with time. The gain drops sharply after $\sim 5$ hours, and reaches a stable value later.

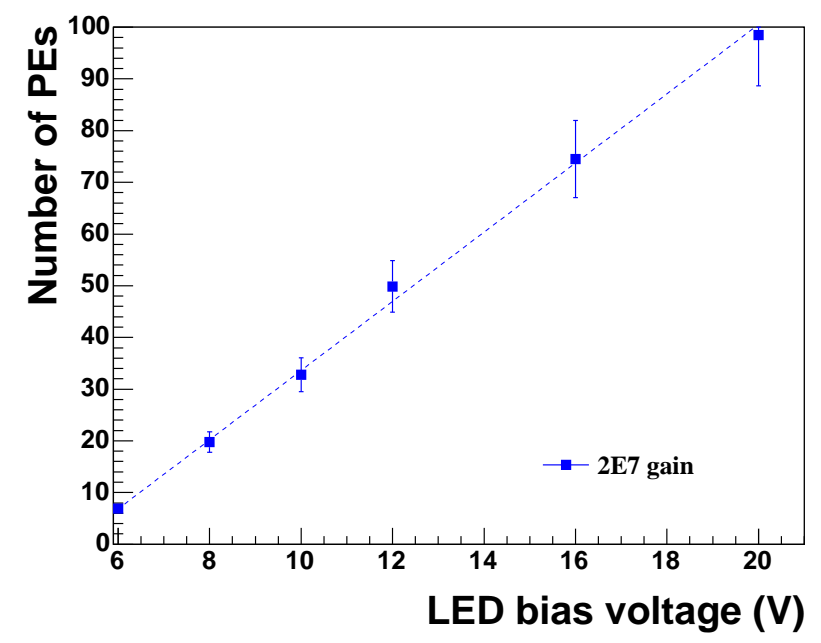

Figure 8. A typical linearity test plot. The number of PEs is measured as a function of LED bias voltage, which affects the light output. Non-linearity would be evident in narrower Poisson distributions at higher light levels, which would make the number of visible PEs higher.

shows a linear dependence on the bias voltage. This LED is then used to measure the number of PEs observed in the PMT as a function of the LED bias voltage. Figure 8 shows a typical result. 

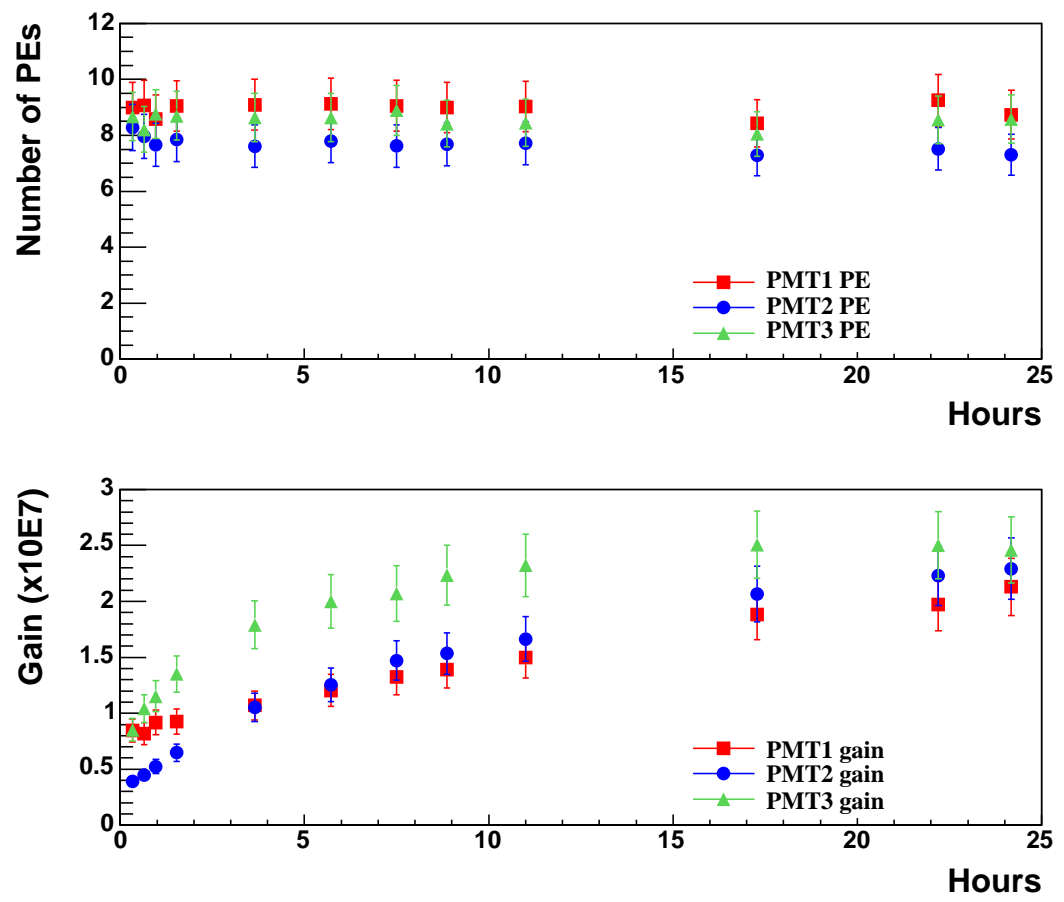

Figure 9. Repeating the stability test with higher LED pulser frequency $(10 \mathrm{kHz}$, instead of $10 \mathrm{~Hz})$. Note that PMT gains change on a long time scale, and it takes $\sim$ one day or more to reach the gain plateau.

The maximum attainable number of PEs for the cryogenic temperature test is around 50 to 100 , Within this range and the expected MicroBooNE PMT gain $\left(\sim 3 \times 10^{7}\right.$, discussed in Sec. 3.2), all tested PMTs show good linearity; this provides a measure of confidence that the MicroBooNE PMTs will perform in a linear fashion up to $~ 100$ PEs in the cryogenic environment.

\subsubsection{Rate dependence of PMT gain}

The PMT rate test shows that a high rate of LED pulses affect tube performance. Figure 9 is the same test as performed in Sec. 3.1.1 (Fig. 6), but in this instance the pulser driving the LED is running at higher frequency $(10 \mathrm{kHz})$. The gains of the PMTs change on a long time scale after being brought to operating voltage. The number of measured PEs is observed to be stable, and as with the previous stability test there appears to be no change in the test environment. The gains increase very slowly, and it takes over a day to reach the plateau. Also this gain plateau is lower than that measured at low frequency.

A second related test examined the effect of changing the LED pulser frequency. Figure 10 is an example of such effect. Here, the LED frequency is changed from $10 \mathrm{~Hz}$ to $10 \mathrm{kHz}$ over 6 hours. The LED frequency is changed 5 times, 10 to 100,100 to 1,000, 1,000 to 2,000, 2,000 to 4,000 then 4,000 to 10,000 in every hour. In this range, the PMT gain is $10-20 \%$ suppressed. Also, when LED frequency is high, PMT gain shows both a long time-scale drift and a hysteresis over the changing of the LED intensity and/or the PMT HV. All rate dependencies of PMT gains are present only for the LN2 tests, and common to all tested PMTs; specific details, however, may depend on the individual characteristics of each PMT. We also note that we do not observe any of 

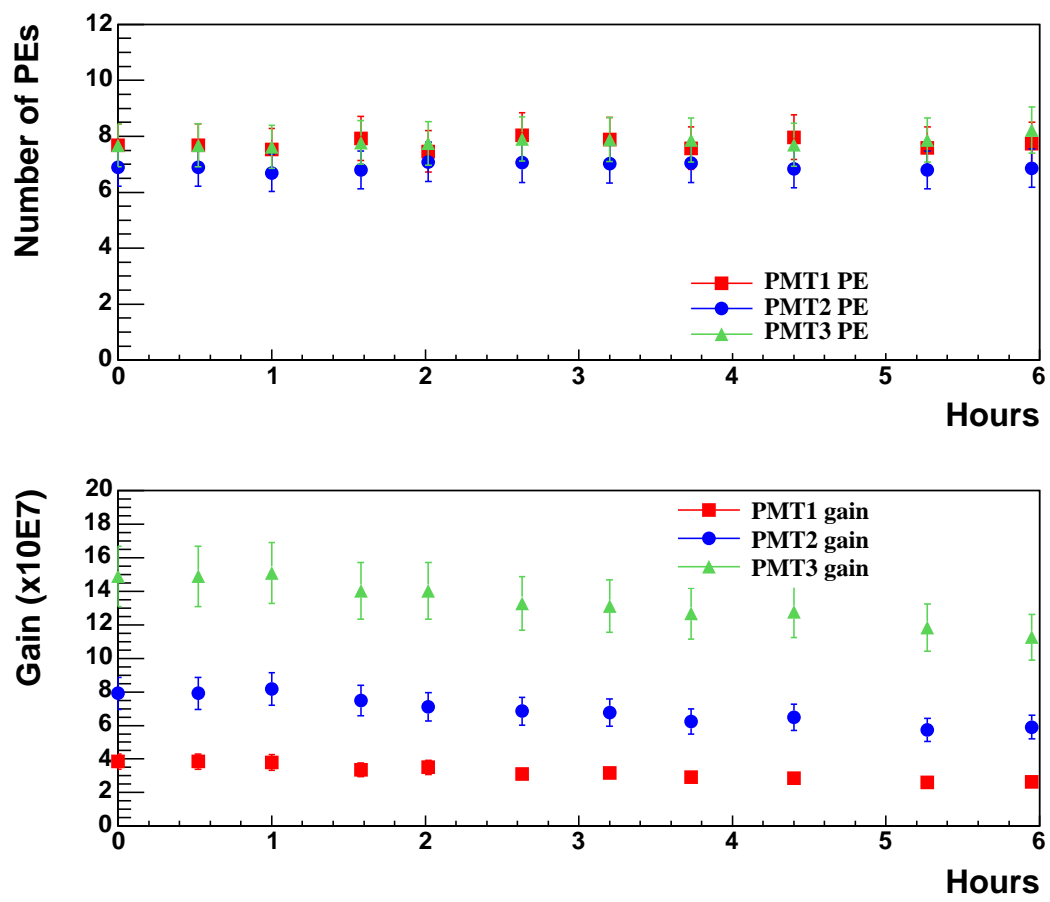

Figure 10. PMT gain drift by changing LED frequency. Here, LED frequency is changed from $10 \mathrm{~Hz}$ to $10 \mathrm{kHz}$ over 6 hours. Note that during data taking period measured PEs are constant for all PMTs.

these phenomena with room temperature running with $10 \mathrm{kHz}$ LED. This is a confirmation that the $10 \mathrm{kHz}$ is not considered the fast signal for our PMT and base. One can speculate that dynodes later in the chain may lack sufficient electrons to respond when the pulse frequency is high in the cold environment due to the low mobility of electrons, but the real cause is presently unknown. This has some implications for PMT operation in a high rate background, such as a LAr detector running on the surface and exposed to cosmic rays. Under such conditions, the PMTs would not achieve their optimal gain, even though the gain would reach a plateau at some point. This test suggests that this optimal plateau may be somewhat unstable, therefore running cryogenic PMTs in high rate environments may require special attention.

\subsection{MicroBooNE PMT gain measurement}

The measurement of PMT gain runs the LED at low intensity (producing 1-2 PEs), allowing the PMT gain to be found from the separation of the single PE peak from the pedestal. This is accomplished with greater accuracy by fitting the single photoelectron response plot, using a procedure described in Ref. [22]. This approach deals correctly with the two types of noise (signal broadening and spurious small signal) and allows absolute calibration. The SER is recorded in $100 \mathrm{~V}$ steps from 900 to $1800 \mathrm{~V}$ and fitting this response allows the gains of all 30 PMTs to be measured, both in air and in LN2. Figure 11 shows the typical fit. The procedure simultaneously fits seven SER parameters, including the location of the pedestal and the single PE peak; the separation of these two parameters measures the PMT gain. 


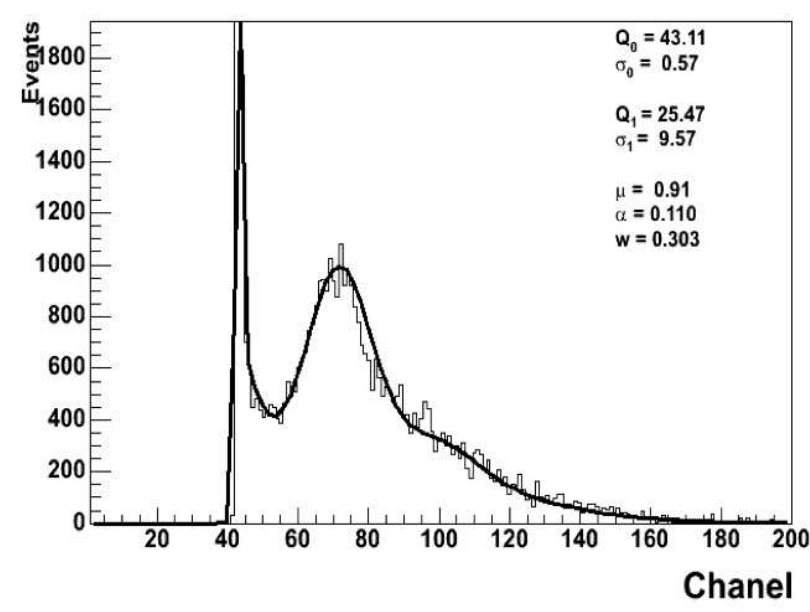

Figure 11. A typical screen shot for the SER fit, performed for all tested PMTs from 900 to $1800 \mathrm{~V}$. The pedestal, single photoelectron peak, and a small two PE peak can be seen. The fit simultaneously finds seven parameters of the single photoelectron response; for details, see Ref. [22].

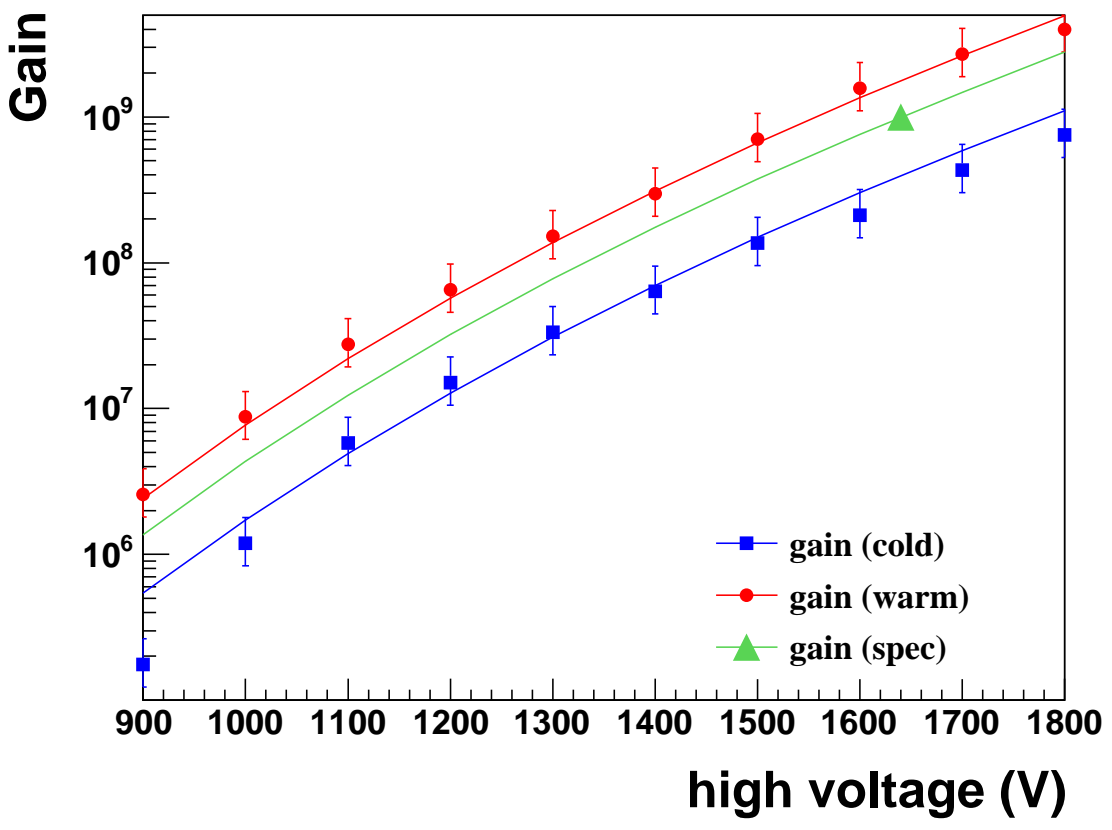

Figure 12. A typical gain plot for a MicroBooNE PMT. All of them are overlaid with 11th power law. The blue square markers are for the LN2 running, the circle markers are for room temperature, and the green triangle marker is from the manufacturer's specification sheet.

The MicroBooNE electronics design requires $\sim 3 \times 10^{7}$ gain, so the one of the goals of the PMT test is to find the HV needed to achieve this gain for each PMT. Figure 12 shows a typical gain of a PMT. The blue square markers are data taken at cryogenic (LN2) temperature, and the red 


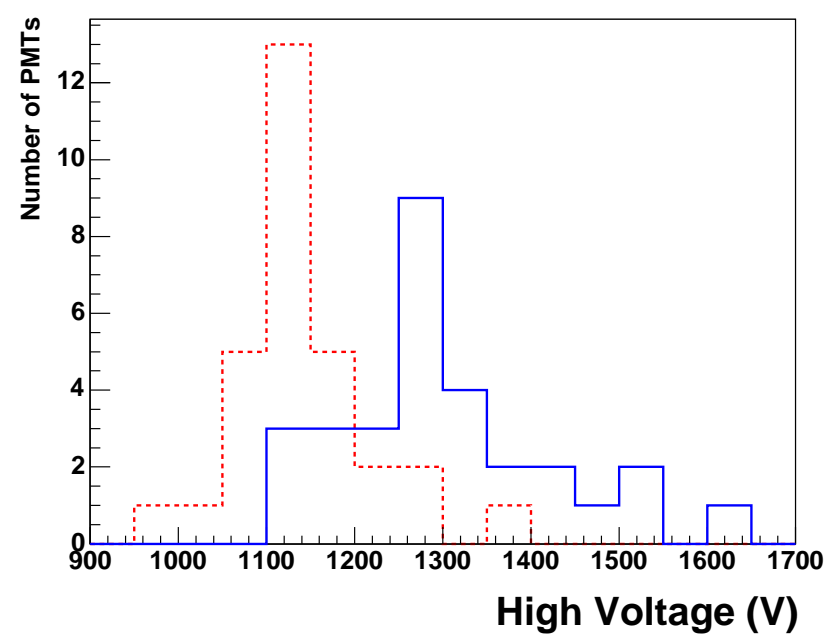

Figure 13. Voltage distributions for the $30 \mathrm{PMTs}$ set to provide $3 \times 10^{7}$ gain. The blue solid histogram id the distribution of the voltages at LN2 temperature, and the red dashed histogram is the distribution of the voltages at room temperature.

circle markers are from the room temperature run. The green triangle markers are for the gain from the manufacturer's specifications sheet. The specifications only provide one HV value to achieve the $10^{9}$ gain, so the rest of the points are extrapolated by assuming that the gain goes as the voltage to the eleventh power; so the rest of the points are extrapolated the exponent is chosen from the data (red and blue curves in Fig. 12). The error is estimated from the multiple measurements made on the calibration PMT. The calibration PMT is fixed at the same location during all tests, and it is immersed in LN2 and warmed up multiple times to monitor changes in the test environment.

The slope of the measured gains can be seen to follow a simple power law, with some variations at the the low and high voltage ends of the distribution. The gain does however follow this power law in the region of interest, where a PMT achieves a $\sim 3 \times 10^{7}$ gain. This allows an operating HV to be extrapolated from neighboring values by fitting to a power law. Most of the PMTs show gains 10 to $30 \%$ higher than the $10^{9}$ at the HV values given in the manufacturer's specifications. The reason for this is presently unknown. Also, as expected, the gain in LN2 is lower than the gain at room temperature. Typically, the cold gain is $\sim 10-50 \%$ of the warm gain. This result is consistent with other measurements [23, 24]. Figure 13 shows the summary of optimal HV values to achieve $3 \times 10^{7}$ gain from all PMTs. It is easy to compensate for the diminution in the PMT gain caused by running at cryogenic temperatures by increasing the tube voltage $\sim 200 \mathrm{~V}$.

\subsection{MicroBooNE PMT dark current measurement}

The dark current is measured for all PMTs in air and for half of the PMTs in LN2. It is known that the dark current at cryogenic temperature is higher than the room temperature for platinum under layered cryogenic PMTs [10, 25, 26]. The dark current is defined as the rate of spontaneous single PE signals without a light source, at a level above a $3 \mathrm{mV}(5 \mathrm{mV})$ threshold for the cold (warm) runs. This is the typical single PE signal height for PMTs when the gains are low. This 


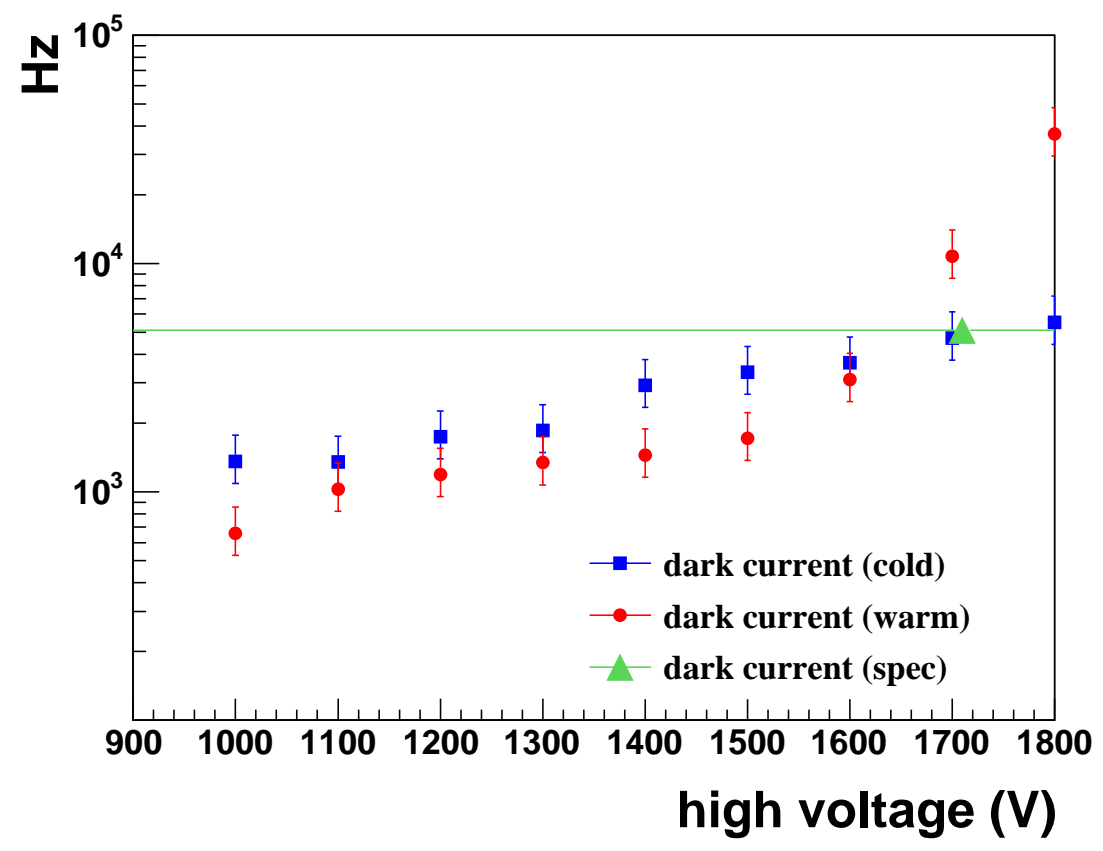

Figure 14. A typical dark current plot for a MicroBooNE PMT. The blue square markers are for the LN2 running, and the red circle markers are for room temperature. The green triangle marker shows the dark current value at $10^{9}$ gain at optimal $\mathrm{HV}$ from the manufacturer's specifications.

definition cannot be rigorously correct, because each PMT has a different single PE pulse height; some PMTs may only barely achieve this threshold when the HV is low, or for high gain PMTs, there may be too many $<1$ PE signals which pass this threshold. Figure 14 shows a typical dark current plot from one PMT. Again, the blue square markers are for the cold run, and the red circle markers are for the warm run. The green triangle marker shows the dark current value from the manufacturer's specifications. As before, the specifications provide only a single point at the HV value where the gain of the PMT achieves $10^{9}$. Not knowing the trend of the dark current values made it impossible to extrapolate additional points. The error is estimated from the variation in the calibration PMT data. The PMT shows a dark current count plateau over a wide range, where the gain increases exponentially, but the dark current increases only linearly. This effect holds true for all PMTs, leading to the above definition of the dark current. Most PMTs show a breakdown of the plateau around 1600-1700 V at room temperature, but all dark current measurements in LN2 show plateau behavior up to $1800 \mathrm{~V}$; this is consistent with Ref. [26].

\section{Conclusion}

This paper reports the testing procedures and results for the MicroBooNE photomultiplier tubes. The PMT base has been designed and tested, bases have soldered to all of the PMTs, and all tubes have been confirmed as working in a cryogenic (LN2) environment. The cryogenic PMT test stand was designed utilizing a large Dewar and was found to provide a test environment stable on a long 
time scale. Several characteristic behaviors of a sample of the cryogenic PMTs were measured, as were gains and dark currents for all PMTs. The PMT gains were found to be lower at cryogenic temperatures, but increasing the tube voltage by $\sim 200 \mathrm{~V}$ compensates for this decrease.

\section{Acknowledgments}

The authors thank the National Science Foundation (NSF-PHY-0847843 and PHY-1000214) for financial support. We thank Yi Chen of the Brookhaven National Laboratory for sharing the fit code, and Stephen Pordes, Linda Bagby, Sten Hansen, Cary Kendziora, William Miner, Kelly Hardin, and Nathan Bremer of the Fermi National Accelerator Laboratory for helping to build the system. We also thank to Flavio Cavanna, Steven Linden, and Vasilli Papavassiliou for their careful readings of this manuscript.

\section{References}

[1] B. J. P. Jones, PoS EPS-HEP2011 (2011) 436, arXiv:1110.1678 [hep-ex].

[2] T. Akiri et al. [LBNE Collaboration], arXiv:1110.6249 [hep-ex].

[3] S. Bertolucci et al., arXiv:1208.0512 [hep-ex].

[4] A. Badertscher et al., arXiv:0804.2111 [hep-ex].

[5] S. Amerio et al., Nucl. Instrum. Meth. A 527 (2004) 329.

[6] T. Doke et al., Nucl. Instrum. Meth. A 291 (1990) 617.

[7] S. Kubota et al., Phys. Rev. B 20 (1979) 3486.

[8] A. Hitachi et al., Phys. Rev. B 27 (1983) 5279.

[9] http://sales.hamamatsu.com/assets/pdf/parts_R/LARGE_AREA_PMT_TPMH1286E05.pdf

[10] H. O. Mayer, Nucl. Instrum. Meth. A 621 (2010) 437.

[11] B. Baptista et al., arXiv:1210.3793 [physics-ins.det].

[12] C. S. Chiu et al., JINST 7 (2012) P07007.

[13] B. J. P. Jones et al., arXiv:1211.7150 [physics-ins.det].

[14] http://amuneal.com/sites/default/files/presentations/shielding/Amuneal_Spotlight_A4K.pdf

[15] E. Calvo et al., Nucl. Instrum. Meth. A 621 (2010) 222.

[16] http://www.rogerscorp.com/documents/726/acm/RO4000-Laminates—Data-sheet.aspx

[17] L. Bugel et al., Nucl. Instrum. Meth. A640 (2011) 69.

[18] http://www.kgw-isotherm.com/produkte/cryo/dss.html

[19] http://www.solidsealing.com/parts/documents/FA13393SL.pdf

[20] http://www.tek.com/oscilloscope/tds5052-manual/memory-erasure-and-nonvolatile-parts-listinstructions

[21] http://css.desy.de/content/index_eng.html

[22] E. H. Bellamy et al., Nucl. Instrum. Meth. A 339 (1994) 468. 
[23] A. Ankowski, Nucl. Instrum. Meth. A 556 (2006) 146.

[24] A. Bueno et al., JINST 3 (2008) P01006.

[25] J. A. Nikkel et al., JINST 2 (2007) P11004.

[26] H. O. Mayer, arXiv:0805.0771 [hep-ex]. 